For personal use only. Not to be reproduced without permission of the publisher (editorial@ gabi-journal.net).

\title{
The authorization of non-biological complex drugs (NBCDs) follow-on versions: specific regulatory and interchangeability rules ahead?
}

\author{
Professor Stefan Mühlebach 1,2, PhD; Professor Arnold Vulto ${ }^{3}$, PharmD, PhD; \\ Jon SB de Vlieger ${ }^{4}$, PhD; Vera Weinstein ${ }^{1,5}$, PhD; Beat Flühmann ${ }^{1,6}$, PhD; \\ Vinod PShah', PhD
}

\begin{abstract}
Introduction: Besides biologicals, a new class of complex drugs - non-biological complex drugs (NBCDs), e.g. liposomes, iron carbohydrate products and glatiramoids - has emerged. Originator NBCD products have been approved by established regulatory rules. However, their follow-on products comprise a challenge to the regulators, manufacturers, physicians and pharmacists.

Methods: An expert panel at a closed workshop during FIP (International Pharmaceutical Federation) Centennial Congress 2012 discussed non-clinical and clinical aspects that distinguish NBCDs from traditional, small molecule drug products as well as new approaches for regulatory evaluation of NBCD follow-on products.

Results: The active 'substance' of an NBCD is of non-biological origin and comprises a heteromolecular mixture of closely related, often nanoparticulate, structures that cannot be fully characterized physicochemically by state-of-the-art analytical means. The composition, quality, and in vivo performance of NBCDs are highly dependent on the manufacturing processes of both the active ingredient and the formulation. Furthermore, pharmacokinetics and pharmacodynamics can be substantially influenced by underlying diseases (particularly in case of associated inflammation). The abridged pathway for regulatory assessment of small molecule generics is not appropriate for NBCD follow-on products whereas the biosimilar pathway is not applicable to nonbiologicals.

Conclusions: New regulatory approaches for the approval of NBCD follow-on products that focus on advanced (analytical) technologies for in vitro characterization and on comparability of clinical safety and therapeutic efficacy are emerging. In contrast to generics, automatic interchange and substitution of NBCD follow-on products should be generally discouraged unless demonstration of therapeutic equivalence and similar safety profiles by appropriate studies.
\end{abstract}

Keywords: Glatiramoids, iron sucrose, liposomes, nanomedicines, NBCD, similars

\section{Introduction}

The progress in pharmaceutical sciences and manufacturing techniques together with more targeted and better-tolerated pharmaceutical formulations gave rise to a new class of medicinal products with complex macromolecular (nanoparticulate) structures, the so-called non-biological complex drugs (NBCDs) [1, 2]. Amongst others, NBCDs comprise liposomes, iron carbohydrate products and glatiramoids. The complexity of these synthetic NBCDs may even exceed that of biologicals. Originator NBCDs have received regulatory approval based on proven quality, efficacy and safety and have been established for many years. Some NBCDs have already been introduced before the growing awareness about the specific issues related to the production and characterization of nanostructures. Accordingly, regulatory approval of NBCD follow-on products is subject to a lively discussion about the requirements to prove therapeutic equivalence and being eligible as substitute for the reference product.

The Non-Biological Complex Drugs Working Group at Dutch Top Institute Pharma, a public-private partnership in The Netherlands, is a network of scientific and clinical experts from academia, industry, regulatory bodies, and knowledge institutes to discuss specific aspects of the development and evaluation of NBCDs and give its expert opinion. This paper briefly summarizes the key discussion points of a closed workshop at the FIP (International Pharmaceutical Federation) Centennial Congress in 2012, organized by the FIP Board of Pharmaceutical Sciences, in order to assist the understanding of the regulatory challenges associated with NBCD follow-on products such as the definition of science-based policies for interchange and substitution. The closed workshop on invitation only was followed by an open discussion symposium the following day at the FIP Centennial Congress about the proposed terminology and a planned paper on points to consider for regulation $[3,4]$.

What are NBCDs and what makes them special?

NBCDs are defined as a medicinal product of non-biological origin with an active substance that is not a homo-molecular structure, but consists of different closely related and mostly nanoparticulate structures. Accordingly, there is not a single substance that can be isolated, quantitated and fully characterized or described by state-of-the-art physicochemical analytical means [4]. Changes in the composition and morphology of an NBCD can substantially influence the quality, biological properties and therapeutic profile of the medicinal product and result from minute variations in the manufacturing process [1, $2,5]$. However, not all structural changes and mechanisms that affect the therapeutic profile are fully understood. Notably, the complexity of NBCD prevents establishing full proof of pharmaceutical equivalence by state-of-the-art analytical means, which comprises one of the two pillars in the evaluation of a generic medicinal product, see Figure 1.

In contrast to the mainly direct and systemic drug-target interaction of small molecules with defined receptors in a

Author for correspondence: Professor Stefan Mühlebach, PhD, Vifor Pharma Ltd, CH-8152 Glattbrugg, Switzerland

Submitted: 12 August 2013; Revised: 24 September 2013; Accepted: 27 September 2013; Published online first: 11 October 2013 
Figure 1: NBCDs: therapeutic equivalence from manufacturing to efficacy and safety

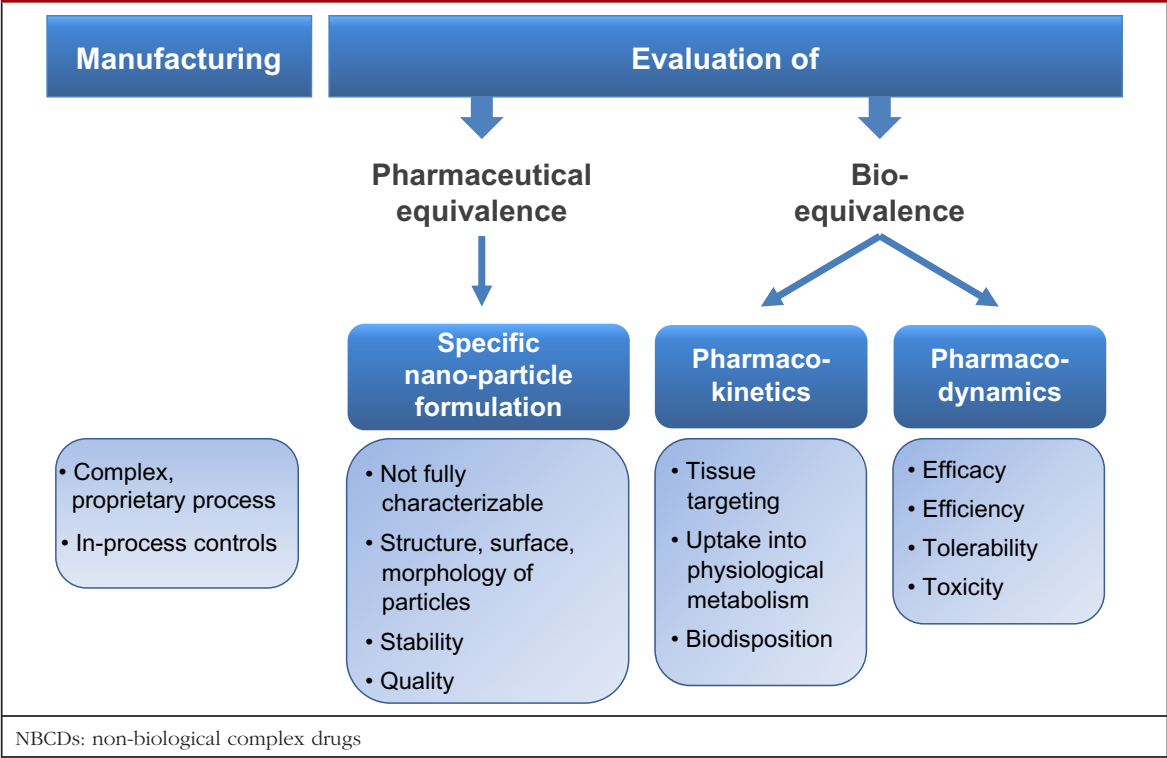

concentration-dependent manner, most NBCDs comprise nanoparticles from which the active ingredient has to be released or formed and then transported to certain biological compartments where the intended activity should be performed. Even a slightly different release or formation rate of the active ingredient, e.g. due to differences in higher order structure of the precursor molecules, can negatively affect the safety and efficacy profile of an NBCD or its follow-on product. For example, in products for intravenous iron therapy such as iron carbohydrate nanoparticles, the highly reactive iron is bound in a polynuclear core, which in turn is stabilized by a carbohydrate shell. After intravenous administration, this complex is first phagocytized by monocytes where the iron is released and transiently stored before it is bound to transferrin and transported to the target tissue where it exerts its action, e.g. incorporation into newly synthesized erythrocytes in the bone marrow; or is stored in an accessible compartment for later physiological use $[6,7]$. Hence, the biological activity of an NBCD is not necessarily correlated to its serum pharmacokinetics (central compartment), the generics pathway's second pillar to show bioequivalence.

In the case of glatiramoids, products that comprise a complex mixture of polypeptides for the treatment of relapsing-remitting multiple sclerosis, even no pharmacokinetic profile and no validated biomarker for efficacy are available. However, although the originator, Copaxone ${ }^{\circledR}$, and a follow-on product are similar in many physiochemical properties, e.g. size distribution, molar ratio of amino acids; sensitive chemical and biological analyses demonstrated differences between those products, e.g. gene expression patterns of glatiramoid-primed murine splenocytes [8].

\section{Does the generics approach fit for NBCD follow-on products and their manufacturers?}

In contrast to follow-on compounds of biologicals that are evaluated according to distinct biosimilar guidelines (originally established in the EU), some NBCD followon products are classified as generics although the two prerequisites for the generics approach cannot be fulfilled. As outlined above, the inability to fully characterize an NBCD prevents proof of pharmaceutical equivalence or clinically meaningful differences between a followon NBCD and its reference product. Moreover, bioequivalence assessment in healthy volunteers does not necessarily reflect the biological fate of and therapeutic response to an NBCD as outlined by the example of the iron sucrose complex below. Notably, this lack in proof of therapeutic equivalence and potential differences in tolerability or safety of NBCD follow-on products can easily become a concern since these products are often used as chronic treatment and in patients with already poor health states, e.g. iron carbohydrate products for haemodialysis patients, liposomal formulations of cytotoxic agents for cancer patients and glatiramoids for multiple sclerosis patients [1, 9, 10]. In such patients, even slight but clinically meaningful differences between the products may interfere with clinical success and thus the feasibility for interchange with the innovator's product.

The potential clinical consequences of the above-mentioned differences could be illustrated by comparative clinical and non-clinical studies of a well-established NBCD (iron sucrose, Venofer $^{\circledR}$ ) and different follow-on preparations (iron sucrose similars, ISS). One study evaluated the effects of switching iron treatment from the iron sucrose originator to an ISS in 75 consecutive stable, haemodialysis-dependent chronic kidney disease patients who underwent at least 60 dialysis sessions before and after the switch at a French dialysis centre [11]. After the switch to an ISS, haemoglobin levels decreased rapidly and anaemia medication had to be increased to return to targeted haemoglobin levels after quite a lengthy re-adjustment period. In addition to this apparent lack of therapeutic equivalence of an ISS, other ISS were associated with an increased risk of adverse events (658 patients at a South Korean centre who had been treated with the iron sucrose originator or an ISS) [12], even if the originator iron sucrose has been well tolerated before (three case reports in Germany) [13]. Furthermore, non-clinical studies showed not only differences between ISS and the originator product [14] but also among different ISS [15], particularly with respect to off-target iron disposition from ISS and induction of oxidative stress and inflammation.

In many countries generics approval of follow-on products allows automatic substitution at the pharmacy level. Since the International Nonproprietary Names of the innovator's and follow-on products are the same, clinicians, caregivers and patients are often not aware of the change in medication. In contrast to the substitutability and interchangeability of fully characterized small molecule generics with well-established therapeutic equivalence, approval for substitution or interchange of NBCD products should only be granted on the basis of appropriate non-clinical and/ or clinical comparisons. Together with the comparability of physicochemical quality this would allow to exclude clinically meaningful differences between the NBCD follow-on products and the reference 
product [1, 2]. Lacking information in case of insufficient response or intolerance can lead to unnecessary diagnostic tests and use of potentially more invasive and more expensive treatment options [8, 10]. Overall, drug product replacement that is guided by acquisition cost only may increase other costs and not be cost-effective from the patient's and payer's perspective.

\section{Workshop discussions favour a similar approach}

At the NBCD workshop in the course of the FIP Centennial Congress 2012, experts from academia, industry, regulatory bodies and knowledge institutes agreed that there is a need for a globally harmonized approach to authorize NBCD follow-on products. This approach should be linked to an accepted common terminology [4]. Also the requirements for an abbreviated procedure showing comparability between different types of NBCD followon products and feasible reference products should be clear. In order to approve an NBCD follow-on product that will be interchangeable with the innovator's product, relevant and comparative clinical and/or non-clinical trials should be performed. Aims of these studies will be an appropriate characterization of the NBCD with up-to-date analytical techniques and to identify the extent of similarity with the originator product. Clinical trials should be sufficiently powered, conducted in patients rather than healthy volunteers to cover for disease-associated changes in pharmacokinetics (PK) and pharmacodynamics (PD). Furthermore, trials should include suitable biological tests to evaluate the similarity of $\mathrm{PK}, \mathrm{PD}$ and safety/tolerability in populations with similar aetiology of the disease as for the aimed indication. For these means also surrogate efficacy and safety markers may be used. Based on the degree of similarity that could be confirmed by the results of such trials and markers, approval of an NBCD follow-on product can be gradually extended to allow for interchange or even automatic substitution in newly diagnosed patients or those on existing chronic treatment. Such a stepwise similarity approach towards totality of evidence can help manufacturers of follow-on products in the development of safe and effective products and to make a realistic prediction of development costs and timelines.

Furthermore, the expert panel indicated that post-approval pharmacovigilance for NBCD and follow-on products should be based on specific brand names as already proposed earlier [16]. An information exchange among treating healthcare professionals and eventually the patient is mandatory to allow for appropriate treatment and drug product traceability in the individual patient.

\section{Conclusions}

An increasing number of NBCDs including nanomedicines become target for development and introduction of followon products. Recent clinical data with NBCD follow-on products (iron sucrose) that were approved like small molecule generics revealed significant differences in efficacy and tolerability compared to the originator product. Accordingly, regulators are prompted to establish a global regulatory framework that considers the structural complexity and specific biological properties of NBCD and provides clear guidance for the development and documentation of safe and effective follow-on products. The experts from academia, industry, regulatory bodies and knowledge institutes at the FIP 2012 workshop suggest a stepwise similarity approach that includes appropriate clinical and/or non-clinical studies that evaluate markers of PK, PD (if applicable) and safety/efficacy in relevant patient populations. As long as proof of therapeutic equivalence and similar safety profiles by appropriate studies is missing, interchange and automatic substitution between NBCDs and their followon products should be discouraged. Overall, a critical review of the current and emerging regulation of NBCDs and NBCD follow-on products encourage further multidisciplinary research and consensus discussions among all stakeholders to develop guidance towards the definition of an NBCD and the development of NBCD follow-on products [3].

\section{Acknowledgement}

Medical writing support was provided by Mr Walter Fürst, SFL Regulatory Affairs and Scientific Communication, Switzerland, and funded by Vifor Pharma Ltd.

Disclosure of financial and competing interests: This manuscript was written within the framework of the Non-Biological Complex Drugs Working Group, hosted at Dutch Top Institute Pharma. The NBCD Working Group is currently supported by Sanofi, Teva Pharmaceutical Industries Ltd and Vifor Pharma International Inc. Professor Stefan Mühlebach is an employee of Vifor Pharma Ltd, Dr Vera Weinstein is an employee of Teva Pharmaceutical Industries, and Dr Beat Flühmann is an employee of Vifor Fresenius Medical Care Renal Pharma.

Provenance and peer review: Not commissioned; externally peer reviewed.

\section{Authors}

Professor Stefan Mühlebach ${ }^{1,2}, \mathrm{PhD}$

Professor Arnold Vulto ${ }^{3}$, PharmD, PhD

Jon SB de Vlieger ${ }^{4}, \mathrm{PhD}$

Vera Weinstein ${ }^{1,5}, \mathrm{PhD}$

Beat Flühmann ${ }^{1,6}, \mathrm{PhD}$

Vinod P Shah ${ }^{1}$, PhD

${ }^{1}$ Steering Committee member, NBCD Working Group, TI Pharma, Leiden, The Netherlands

${ }^{2}$ Vifor Pharma Ltd, Glattbrugg, Switzerland ${ }^{3}$ Erasmus University Medical Center, Hospital Pharmacy, Rotterdam, The Netherlands ${ }^{4}$ TI Pharma, PO Box 142, NL-2300 AC Leiden, The Netherlands

${ }^{5}$ Teva Pharmaceutical Industries, Petach Tikva, Israel

${ }^{6}$ Vifor Fresenius Medical Care Renal Pharma, St Gallen, Switzerland

\section{References}

1. Borchard G, Fluhmann B, Muhlebach S. Nanoparticle iron medicinal products-requirements for approval of intended copies of non-biological complex drugs (NBCD) and the importance of clinical comparative studies. Regul Toxicol Pharmacol. 2012;64(2):324-8.

2. Schellekens H, Klinger E, Mühlebach S, et al. The therapeutic equivalence of complex drugs. Regul Toxicol Pharmacol. 2011;59(1):176-83.

3. Schellekens H, Stegemann S, Weinstein V, et al. How to regulate nonbiological complex drugs (NBCD) and their follow-on versions: points to consider. AAPS J. 2013 Sep 25. doi:10.1208/ s12248-013-9533-Z

4. Crommelin DJ, de Vlieger JS, Weinstein V, et al. Different pharmaceutical products need similar terminology. AAPS J. 2013 Sep 25. doi:10.1208/ s12248-013-9532-0

5. Ehmann F, Sakai-Kato K, Duncan R, et al. Nextgeneration nanomedicines and nanosimilars: EU regulators' initiatives relating to the development and evaluation of nanomedicines. Nanomedicine (Lond). 2013;8(5):849-56.

6. Evstatiev R, Gasche C. Iron sensing and signalling. Gut. 2012;61(6):933-52.

7. Geisser P, Burckhardt S. The pharmacokinetics and pharmacodynamics of iron preparations. Pharmaceutics. 2011;3(1):12-33.

8. Bakshi S, Chalifa-Caspi V, Plaschkes I, et al. Gene expression analysis reveals functional pathways of glatiramer acetate activation. Expert Opin Ther Targets. 2013;17(4):351-62. 
9. Mamidi RN, Weng S, Stellar S, et al. Pharmacokinetics, efficacy and toxicity of different pegylated liposomal doxorubicin formulations in preclinical models: is a conventional bioequivalence approach sufficient to ensure therapeutic equivalence of pegylated liposomal doxorubicin products? Cancer Chemother Pharmacol. 2010;66(6):1173-84.

10. Varkony $\mathrm{H}$, Weinstein $\mathrm{V}$, Klinger $\mathrm{E}$, et al. The glatiramoid class of immunomodulator drugs. Expert Opin Pharmacother. 2009;10(4):657-68.

11. Rottembourg J, Kadri A, Leonard E, et al. Do two intravenous iron sucrose preparations have the same efficacy? Nephrol Dial Transplant. 2011;26(10):3262-7.

12. Lee ES, Park BR, Kim JS, et al. Comparison of adverse event profile of intravenous iron sucrose and iron sucrose similar in postpartum and gynecologic operative patients. Curr Med Res Opin. 2013;29(2):141-7.

13. Stein J, Dignass A, Chow KU. Clinical case reports raise doubts about the therapeutic equivalence of an iron sucrose similar preparation compared with iron sucrose originator. Curr Med Res Opin. 2012;28(2):241-3.

14. Toblli JE, Cao G, Oliveri L, et al. Comparison of oxidative stress and inflammation induced by different intravenous iron sucrose similar preparations in a rat model. Inflamm Allergy Drug Targets. 2012;11(1):66-78.

15. Toblli JE, Cao G, Giani J, et al. Different effects of European iron sucrose similar preparations and originator iron sucrose on nitrosative stress, apoptosis, oxidative stress, biochemical and inflammatory markers in rats. NDT Plus 2011;4 [abstract]:SuO028.

16. Wysowski DK, Swartz L, Borders-Hemphill BV, et al. Use of parenteral iron products and serious anaphylactic-type reactions. Am J Hematol. 2010;85(9):650-4. DOI: 10.5639/gabij.2013.0204.054

Copyright (c) 2013 Pro Pharma Communications International 